\title{
Section B:
}

\section{Structure \& Atmospheres}




\title{
Alien Earth: Glint observations of a remote planet
}

\author{
Richard K. Barry ${ }^{1}$ and L. Drake Deming ${ }^{1}$ \\ ${ }^{1}$ National Aeronautics and Space Administration \\ Goddard Space Flight Center, Greenbelt, MD \\ email: Richard.K.Barry@NASA.gov
}

\begin{abstract}
We give a preliminary report on a multi-wavelength study of specular reflections from the oceans and clouds of Earth. We use space-borne observations from a distance sufficient to ensure that light rays reflected from all parts of Earth are closely parallel, as they will be when studying exoplanets. We find that the glint properties of Earth in this far-field vantage point are surprising - in the sense that some of the brightest reflections are not from conventional ocean-glints, but appear to arise from cirrus cloud crystals. The Earth observations discussed here were acquired with the High Resolution Instrument (HRI) - a $0.3 \mathrm{~m} \mathrm{f} / 35$ telescope on the Deep Impact (DI) spacecraft during the Extrasolar Planet Observation and Characterization (EPOCh) investigation.
\end{abstract}

Keywords. infrared: planetary systems, astrobiology, astrochemistry, planetary systems, Earth

\section{Observations}

While EPOCh has as its main scientific goal the observation of transits of exoplanets across the face of their host stars, observations of Earth were also conducted during orbital flyby opportunities. The HRI, used for these observations, has nine filters, seven of which are $100 \mathrm{~nm}$ wide, boxcar bandpass filters centered at 350, 450, 550, 650, 750, 850 and $950 \mathrm{~nm}$. Two other filters are uncoated fused silica with no band limiting, transparent longwards of $700 \mathrm{~nm}$. DI also has an NIR spectrometer that covers the wavelength range from 1.05-4.5 microns with a spectral resolution exceeding 200 over the entire pass band [3]. Using the HRI instrument, Earth was observed on five occasions: 2008-Mar-18 18:18 UT, 2008-May-28 20:05 UT, 2008-Jun-4 16:57 UT, 2009-Mar-27 16:19 and 2009-Oct4 09:37 UT. Each set of observations was conducted over a full 24-hour rotation of Earth and a total of thirteen NIR spectra were taken on two-hour intervals during each observing period. Photometry in the 450, 550, 650 and $850 \mathrm{~nm}$ filters was taken every fifteen minutes and every hour for the 350, 750 and $950 \mathrm{~nm}$ filters.

\section{Data and Discussion}

Glints were located to the greatest precision allowed by the data using the images that had been deconvolved with a PSF previously developed using a drizzle process (Barry et al. 2010). Once the location of the glint events were noted using the deconvolved images, we constructed spectra of the glints from the un-deconvolved images by centering the 8-pixel patches on the locations found in the deconvolved set. Using the process for extracting glint flux and background flux noted above, the un-deconvolved images were used to report these fluxes for purposes of comparison to the theoretical values. These measured values are given in Table 1. 
Table 1. Summary of glint and background blue (450 micron) to IR ( 850 micron) flux ratios for non-deconvolved Earth observations during the EPOXI science investigation. Individual glints are listed as Glint Event North or South (GEN or GES) together with the event's latitude and longitude. The final column gives the assessment of what is in the 8-pixel photometric aperture for the images deconvolved with the PSF.

\begin{tabular}{lccccl}
\hline Event & Longitude & Latitude & Glint B/IR & Bkg B/IR & Observed \\
\hline GEN1 & -159 & 40 & 1.4 & 1.9 & Cloud/Ocean \\
GEN2 & -140 & 40 & 1.1 & 1.8 & Ocean/Cloud \\
GEN3 & -121 & 40 & 1.2 & 1.5 & Land \\
GEN4 & 24 & 40 & 1.0 & 1.3 & Aegean/Mediterranean Seas \\
GEN5 & 54 & 40 & 1.0 & 1.5 & Caspian Sea \\
GEN6 & 66 & 40 & 1.0 & 1.3 & Land \\
GEN7 & 77 & 40 & 1.5 & 1.7 & Cloud/Land \\
GEN8 & 107 & 40 & 1.1 & 1.4 & Land \\
GEN9 & 118 & 40 & 0.3 & 1.7 & Land/Cloud (Bohia Sea) \\
GEN10 & 126 & 40 & 1.0 & 1.7 & Seas around Korea \\
GES1 & -170 & -45 & 1.6 & 1.9 & Cloud \\
GES2 & -38 & -45 & 1.1 & 1.8 & Ocean/Cloud \\
GES3 & -31 & -45 & 1.3 & 1.9 & Ocean/Cloud \\
GES4 & 11 & -45 & 1.6 & 1.9 & Cloud \\
GES5 & 74 & -45 & 2.7 & 1.9 & Cloud \\
GES6 & 168 & -45 & 1.0 & 1.7 & Land \\
\hline
\end{tabular}

Glint Event 1 in the Northern hemisphere (GEN1) together with Glint Events GEN2, GES2, GES3 are unambiguously identified with open ocean and cloud in roughly equal proportion. These are listed in Table 1 together with the comment cloud/ocean or ocean/cloud depending on which hydrological state appeared to dominate the photometric aperture during that observation. GEN3, GEN6, GEN8, and GES6 are all over land with little or no cloud in the photometric aperture. GEN4, GEN5, and GEN10 are all glints from bodies of water that are partially isolated from other, larger bodies of water by land. GEN7 and GEN9 appear to be associated primarily with a combination of land and cloud. Finally, GES1, GES4 and GES5 appear to be glints in photometric apertures dominated by a layer of cloud suggesting a detection of the subsun phenomenon - reflection from flat ice crystals. Referring to Table 1, strong b/I ratio of glint suggests a possible longer passage through atmosphere and consequently more red and IR scattered out by Raleigh scattering. Analysis of these data are ongoing.

\section{References}

Barry, R. K., Lindler, D., Deming, L. D., A'Hearn, M. F., Ballard, S., Carcich, B., Charbonneau, D., Christiansen, J., Hewagama, T., McFadden, L., \& Wellnitz, D. 2010, Space Telescopes and Instrumentation 2010, 7731, 107 